\title{
Editorial
}

Jean-François Hardy MD, Sylvain Bélisle MD, Francine Décary MD PhD

\section{Cardiac surgical patients must not be denied the benefits of autologous blood predonation}

Before the advent of acquired immunodeficiency syndrome (AIDS), transfusion of homologous blood products (HBP) was felt to be a safe procedure, entailing minimal risks to patients inasmuch as $A B O / R h$ incompatibility was avoided. In fact, the major problem faced by blood banks was to keep up with the ever-increasing demand for blood products generated by the growing complexity of the surgical procedures performed such as hepatic transplantation and cardiac surgery. The advent of AIDS dramatically modified this perception of the safety of blood, both for physicians and patients, and has resulted in major changes in the utilization of blood products since 1985 . In 1994, physicians are expected to implement strategies that will decrease or eliminate the need for transfusion of HBP. ${ }^{1}$

These strategies commence in the preoperative period, extend throughout the intraoperative and postoperative periods, and involve (but are not limited to) measures such as an informed consent for transfusion, pharmacological agents to reduce bleeding, tolerance of low haemoglobin concentrations and autologous transfusions.' While intraoperative donation of autologous blood (normovolaemic haemodilution) and intra- and postoperative retransfusion of shed blood have been used for some time in adult cardiac surgery, predonation of autologous blood is, unfortunately, limited to a small number of centres in North America. ${ }^{2}$ Several reasons help to explain this situation, particularly in Canada.

Predonation of autologous blood by "healthy" patients is performed outside hospital, at Canadian Red Cross Blood Centre permanent clinic sites, where direct medical supervision and elaborate equipment are not readily available. Decreasing the oxygen-carrying capacity of

From the Department of Anesthesia, Montreal Heart Institute and the Canadian Red Cross Society Blood Centre, Montreal, Quebec, Canada. blood of patients known to have cardiac disease has always been considered dangerous. Therefore, in-hospital predonation clinics have to be setup for these patients, with the costs, allocation of space and resources, etc., that this entails. In the USA, the acquisition cost of a unit of autologous blood is, on average, $50 \%$ higher than that of a unit of homologous red cells. ${ }^{3}$

The logistics of predonation are not simple. Presently, the shelf life of red blood cells (RBC) is 35 days at $1^{\circ}-6^{\circ} \mathrm{C}$, but $\mathrm{RBC}$ with $100 \mathrm{ml}$ of the additive AS-3 (Nutricel( $\left.{ }^{\circledR}\right)$ have the potential for 42 days shelf life. ${ }^{4}$ This finite period of conservation is a major constraint. Operating schedules must be adhered to strictly in order to avoid wastage of predonated units. On the other hand, enough time must be available to harvest sufficient autologous blood for the proposed procedure. In some cardiac surgical centres, especially in the USA, the delay before the operation is often too short to allow effective predonation. ${ }^{5}$ Finally, the blood bank must ensure that all predonated units are used before HBP are administered.

Even when the predonation clinic exists and the logistics have been smoothed out, patients may be unable to donate, mainly because of anaemia, but also because of difficulties with venous access, intercurrent illness (upper respiratory tract infections most frequently) or various other, less frequent, conditions. ${ }^{6}$ In the study by Kruskall et al. ${ }^{6}$ the haematocrit of patients who predonated a mean of 3.1 units before cardiac surgery decreased by $4 \%$. Most authors ${ }^{7-10}$ consider patients eligible for predonation if their haematocrit is $\geq 34 \%$, but this figure remains somewhat arbitrary. The majority of reactions at the time of predonation are vasovagal and occur in $5 \%$ of healthy donors. ${ }^{9,10}$ Cardiac patients appear to behave similarly, with few untoward events related to their cardiac disease. ${ }^{6-8,11}$ No serious reactions following phlebotomy were apparent in any of the "high-risk" patients 
donating autologous blood in the study by Mann et al. ${ }^{12}$ Nonetheless, close monitoring during and after blood withdrawal by Spiess et al. revealed a number of haemodynamic abnormalities, the importance of which is uncertain since these events did not lead to increased morbidity or mortality. ${ }^{10}$

In this issue of the Canadian Journal of Anaesthesia, Pinkerton describes his preliminary experience with autologous blood predonation in support of cardiac surgery. ${ }^{13}$ In light of the obstacles to predonation in cardiac patients, he must be commended for instituting this hospital-based autologous donor programme since 1991 at Sunnybrook Health Science Centre. The author concludes that autologous blood can make a considerable contribution to transfusion support of cardiac surgery, that two units suffice for $75-80 \%$ of eligible patients and that the use of non-red cell allogenic products requires careful scrutiny if the full benefits of autologous donation are to be realized.

These preliminary results are consistent with previously published studies, ${ }^{7,8,11}$ as discussed by Pinkerton. At the Montreal Heart Institute, approximately $75 \%$ of our first time, elective coronary artery bypass patients are exposed to fewer than three units of RBC. The other $25 \%$ comprises mainly patients receiving HBP to correct a severe coagulation abnormality. While predonation can only be expected to decrease transfusion of HBP in the latter, autologous blood will eliminate the use of HBP entirely in the former. Some centres fractionate predonated blood into RBC and fresh frozen plasma (FFP). The availability of FFP may help reduce further the need for HBP after cardiac surgery. Nonetheless, with or without fractionation, there can be no doubt that predonation is effective in this patient population.

The issue of the optimal quantity of blood to be harvested has not been resolved. At present, blood collected under an autologous programme cannot, in Canada, be redirected for use in other patients ("crossed-over") if it is not required for its donor. ${ }^{4}$ Unused blood is, unfortunately, wasted blood. As pointed out by Pinkerton, sufficient autologous blood must be collected to eliminate the use of HBP in a high percentage of donors while avoiding collection of units that will be wasted, along with the time, efforts and money that go into the process. The optimal number of units will vary according to the procedure to be performed since complex surgery and reoperation require more blood, ${ }^{14}$ and also is determined by the other measures utilized to decrease blood loss. For example, Axelrod et al determined that five autologous units would be necessary to avoid any homologous blood in $90 \%$ of their patients undergoing myocardial revascularization. ${ }^{15}$ At the University of Ottawa Heart Institute, three autologous units will prevent allogenic ex- posure in $80 \%$ of patients, while four units will avoid exposure in $90 \%$ of patients. ${ }^{*}$ Therefore, each programme will have to determine the optimal number of units to be collected for a given patient population.

Recombinant human erythropoietin is being used currently to treat patients with renal failure and other diseases that result in chronic anaemia. In patients about to undergo elective orthopaedic interventions, preoperative administration of erythropoietin increased the ability to donate autologous blood from a mean 4.1 units to 5.4 units. ${ }^{16}$ In combination with autologous blood predonation, erythropoietin reduces transfusion of HBP in patients undergoing cardiac operations ${ }^{17}$ but it is expensive and side effects such as hypertension and hyperviscosity are of concern. It has been recommended that its use be limited to patients who are anaemic before donation (haematocrit $<36 \%$ ) or who are of low body weight $(<52 \mathrm{~kg}) .{ }^{18}$ This appears reasonable, especially in view of the optimal number of units to be collected.

Despite all efforts to reduce blood loss, transfusion of HBP may become necessary despite predonation of autologous blood. In order to decrease the need for HBP, those who order transfusions should do so mindfully rather than automatically, in response to a given pathophysiological trigger. This is particularly true for the administration of RBC: the indications for the transfusion of erythrocytes must be examined closely and the effectiveness of therapy demonstrated. ${ }^{1}$ Attention to a set of arbitrary criteria rather than to the patients' needs explains the great variability of transfusion practice in coronary bypass surgery. ${ }^{2,19}$ All those who order transfusions must be educated about the indications and risks involved. In particular, the prophylactic transfusion of homologous plasma and platelets is of no benefit, carries an unnecessary risk to the patient and must be abandoned. ${ }^{19}$

In the past, the organization of a hospital-based predonation programme for cardiac surgical patients may not have been simple but minds are changing. Such a programme is being instituted at the Montreal Heart Institute, thanks to the intense and enthusiastic cooperation between the Canadian Red Cross Blood Centre in Montreal and all the personnel involved throughout the hospital. Difficulties were encountered but rapidly surmounted, no doubt because of the ever-present concern about the infectious risks of HBP.

In conformity with the recommendations of the $\mathrm{Ca}$ nadian Red Cross Society, a positive serological test ex-

* Robblee $J$. Autologous transfusion, in Transfusion Horizons 94, a symposium presented by the Cardiovascular and Thoracic Section of the Canadian Anaesthetists' Society, Edmonton Convention Centre, June 21, 1994. 
cludes patients from predonating. ${ }^{4}$ This increases the safety of the blood inventory and that of health care personnel but, unfortunately, exposes these patients to the risks of transfusion-transmissible diseases other than the one they carry. ${ }^{9}$ This issue cannot be separated from the problem of obligatory wastage of unused autologous units. Should society decide that unused autologous units can be "carried-over" for homologous use, the risk that an "innocent" recipient is adversely affected by an infected, previously autologous unit will have to be weighed against the risk that patients denied predonation acquire a new transfusion-transmitted disease. ${ }^{20}$

In conclusion, autologous blood predonation in cardiac surgery is a safe, effective and underused means of reducing exposure to HBP while increasing existing blood supplies. A number of peripheral issues remain to be resolved but predonation, in combination with other interventions known to decrease bleeding and transfusions after cardiopulmonary bypass, must become an integral part of the transfusion strategy of centres where cardiac surgery is performed.

\section{Les avantages du sang autologue ne doivent pas être refusés aux malades de chirurgie cardiaque}

Avant la survenue du syndrome d'immunodéficience acquise (SIDA), la transfusion de produits sanguins homologues (PSH) était considérée sécuritaire et peu risquée pour les patients, en autant que l'incompatibilité $\mathrm{ABO} /$ $\mathrm{Rh}$ était évitée. En fait, le problème majeur auquel devaient faire face les banques de sang était de maintenir un approvisionnement suffisant face à la demande croissante de PSH, demande générée par la complexité grandissante des procédures chirurgicales, la transplantation hépatique et la chirurgie cardiaque, par exemple. L'arrivée du SIDA a complètement modifié cette perception de la sécurité des produits sanguins, à la fois pour les médecins et pour les patients, et a entraîné des modifications majeures dans l'utilisation des PSH depuis 1985. En 1994, les médecins doivent mettre en oeuvre tous les moyens à leur disposition pour diminuer ou éliminer complètement les transfusions de PSH.'

Ces stratégies sont mises en oeuvre dès la période préopératoire, couvrent les périodes intra- et postopératoires et comprennent (mais ne sont pas limitées à) des mesures telles que le consentement éclairé avant la transfusion, les approches pharmacologiques pour diminuer le saignement, la tolérance d'une hémoglobine basse et les transfusions autologues. ${ }^{1}$ En Amérique du Nord, la collecte intra-opératoire de sang autologue (hémodilution normovolémique) et la retransfusion intra- et postopératoire du sang perdu sont déjà utilisées en chirurgie cardiaque de l'adulte. Malheureusement, la prédonation de sang autologue n'est disponible que dans quelques centres. ${ }^{2} \mathrm{Plu}-$ sieurs raisons peuvent expliquer cet état de fait, surtout au Canada.

Le don de sang autologue par les patients " en bonne santé " se fait hors du milieu hospitalier, dans les cliniques permanentes des services transfusionnels de la Société canadienne de la Croix-Rouge, là où la supervision médicale et le matériel disponible sont limités. La diminution de la capacité de transport de l'oxygène secondaire à l'anémie a toujours été considérée dangereuse chez les patients porteurs d'une pathologie cardiaque. C'est pourquoi ils ne sont pas admis dans ces cliniques. Des cliniques de prélèvement doivent donc être organisées en milieu hospitalier (i.e., là où les complications peuvent être traitées rapidement) avec les coûts, les locaux, les ressources, etc., que de telles installations exigent. Aux États-Unis, le coût d'acquisition d'une unité de sang autologue est, en moyenne, $50 \%$ plus élevé que celui d'une unité de globules rouges (GR) homologues. ${ }^{3}$

L'organisation d'un programme de don autologue n'est pas chose simple. En ce moment, la durée de conservation des GR est de 35 jours à une température de $1^{\circ}-6^{\circ} \mathrm{C}$, mais les GR additionnés de $100 \mathrm{ml}$ de AS-3 (Nutricel ${ }^{\circledR}$ ) peuvent être conservés pendant 42 jours. ${ }^{4}$ Cette durée de conservation limitée est une contrainte majeure. Les programmes opératoires doivent être respectés méticuleusement, de façon à éviter la péremption des unités autologues. D'autre part, une période de temps assez longue doit être disponible avant l'intervention pour permettre la collecte d'un nombre suffisant d'unités autologues en vue de la chirurgie proposée. Dans certains centres de chirurgie cardiaque, aux États-Unis surtout, le délai avant l'opération est souvent trop court pour permettre une prédonation efficace. ${ }^{5}$ Enfin, la banque de sang doit s'assurer que toutes les unités autologues ont été utilisées avant que des PSH ne soient administrés aux patients.

Même lorsque la clinique de don autologue a été mise sur pied et que les difficultés logistiques ont été aplanies, la collecte de sang peut être impossible, le plus souvent 
en raison d'une anémie, mais aussi à cause de difficultés avec l'accès veineux, d'une pathologie intercurrente telle qu'une infection des voies respiratoires supérieures (surtout) ou diverses autres pathologies, moins fréquentes. ${ }^{6}$ Ainsi, le prélèvement de 3,1 unités en moyenne avant la chirurgie cardiaque a fait chuter l'hématocrite de $4 \%$ dans l'étude de Kruskall et al. ${ }^{6}$ Plusieurs auteurs ${ }^{7-10}$ considèrent qu'un hématocrite $\geq 34 \%$ est adéquat en vue de la prédonation, mais ce chiffre demeure quelque peu arbitraire. Lors de la collecte de sang, la majorité des complications sont d'origine vasovagale, survenant chez approximativement $5 \%$ des donneurs sains. ${ }^{9,10}$ Les patients cardiaques semblent se comporter de façon sembable, avec peu de morbidité reliée à leur pathologie cardiaque même. ${ }^{6-8,11}$ Mann et al. n'ont mis en évidence aucune complication sérieuse secondaire à la phlébotomie chez leurs patients "à haut risque " lors de la collecte de sang autologue. ${ }^{12}$ Néanmoins, un monitorage serré de ces patients pendant et après la saignée par Spiess et al. a révélé un certain nombre d'anomalies hémodynamiques dont la signification demeure incertaine, étant donné l'absence de morbidité ou de mortalité accrue imputable à leurs observations. ${ }^{10}$

Dans ce numéro du Journal Canadien d'Anesthésie, Pinkerton décrit son expérience préliminaire avec le don de sang autologue en chirurgie cardiaque. ${ }^{13} \mathrm{~A}$ la lumière des embûches à la prédonation chez les malades cardiaques, il doit être félicité pour avoir mis sur pied un programme hospitalier de don de sang autologue depuis 1991 au Sunnybrook Health Science Centre. L'auteur conclut que le sang autologue peut apporter une contribution significative aux besoins transfusionnels des patients en chirurgie cardiaque, que deux unités suffisent pour 75 à $80 \%$ des patients éligibles et que l'utilisation des produits sanguins allogènes autres que les GR doit être surveillée attentivement si l'on veut exploiter au maximum les bénéfices du don autologue.

Tel que discuté par Pinkerton, ses résultats préliminaires sont en accord avec les études publiées antérieurement. ${ }^{7,8,11} \mathrm{De}$ façon semblable, à l'Institut de Cardiologie de Montréal, nous avons documenté qu'environ $75 \%$ de nos patients subissant une chirurgie coronarienne élective de première intention sont exposés à moins de trois unités de GR. L'autre 25\% est composé, en majeure partie, de patients recevant des PSH en vue de corriger une sérieuse anomalie de la coagulation. Alors que la prédonation ne pourra que diminuer la transfusion de PSH chez ces derniers, le sang autologue est en mesure d'éliminer totalement l'utilisation de PSH chez les premiers. Certains centres procèdent au fractionnement du sang autologue en GR et en plasma frais congelé (PFC). La disponibilité de $\mathrm{PFC}$ pourra, sans doute, aider à diminuer encore plus le recours aux PSH après la chirurgie car- diaque. Quoi qu'il en soit, avec ou sans fractionnement, il ny a aucun doute que le don autologue est efficace chez ces patients.

La question de la quantité optimale de sang à prélever n'a pas été encore entièrement résolue. En ce moment, au Canada, le sang autologue ne peut être utilisé à des fins homologues si le donneur autologue n'en a plus besoin. ${ }^{4}$ Donc, une unité de sang inutilisée est, malheureusement, une unité de sang gaspillée. Pinkerton souligne à juste titre qu'assez de sang doit être prélevé pour éliminer l'utilisation des PSH chez un pourcentage élevé de donneurs, tout en évitant la collecte d'unités superflues et le gaspillage de temps, de travail et d'argent nécessaires au prélèvement. Le nombre d'unités optimal variera en fonction de la procédure envisagée, les chirurgies complexes et les réinterventions requérant plus de produits sanguins. ${ }^{14}$ La quantité optimale variera également en fonction des autres mesures utilisées pour diminuer les pertes sanguines. Par exemple, Axelrod et al. ont déterminé que cinq unités autologues permettraient d'éviter l'utilisation de PSH chez $90 \%$ de leurs patients subissant une chirurgie de revascularisation myocardique. ${ }^{\text {is }}$ À l'Institut de Cardiologie d'Ottawa, trois unités autologues préviendront l'exposition aux PSH chez $80 \%$ des patients, alors que quatre unités la préviendront chez $90 \%$ des patients. ${ }^{*}$ Ainsi, chaque programme de don autologue aura à déterminer le nombre d'unités à prélever pour une population donnée.

En ce moment, l'érythropoḯtine humaine recombinante est utilisée pour traiter les insuffisants rénaux et les autres pathologies responsables d'une anémie chronique. L'administration préopératoire d'érythropoḯtine a permis d'augmenter le don autologue d'une moyenne de 4,1 unités à une moyenne de 5,4 unités chez les patients subissant une chirurgie orthopédique élective. ${ }^{16}$ L'utilisation d'érythropoiétine, en combinaison avec le sang autologue, diminue les transfusions de PSH chez les patients subissant une chirurgie cardiaque. ${ }^{17}$ Cependant, l'érythropoïetine est coûteuse et les effets secondaires, tels que l'hypertension et l'hyperviscosité, sont à craindre. Il a donc été recommandé que son utilisation soit limitée aux patients anémiques avant le don (hématocrite $<36 \%$ ) ou chez ceux de faible poids $(<52 \mathrm{~kg}) .^{18} \dot{A}$ la lumière de la discussion qui précède sur le nombre dunités optimal à prélever, cette recommandation nous apparaît raisonnable.

Malgré tous les efforts mis en oeuvre pour réduire les pertes sanguines, la transfusion de PSH peut devenir

* Robblee $J$. Autologous transfusion, in Transfusion Horizons 94, a symposium presented by the Cardiovascular and Thoracic Section of the Canadian Anaesthestists' Society, Edmonton Convention Centre, June 21, 1994. 
nécessaire malgré la disponibilité de sang autologue. En vue de diminuer les besoins de PSH, ceux qui prescrivent les transfusions ne devraient le faire qu'après mûre réflexion, évitant de réagir comme des automates à une gâchette transfusionnelle prédéterminée. Ceci est particulièrement vrai de l'administration de GR. Les indications de la transfusion érythrocytaire doivent être examinées avec attention et l'efficacité du traitement devra être documentée. ${ }^{1}$ L'attention portée à un ensemble de critères arbitraires plutôt qu'aux besoins du patient explique la grande variabilité de la pratique transfusionnelle en chirurgie cardiaque. ${ }^{2,19}$ Tous ceux qui prescrivent des transfusions doivent en connaitre parfaitement les indications et les risques. Dans ce contexte, rappelons que la transfusion prophylactique de PFC homologue et de plaquettes est inefficace, expose le malade à un risque inutile et doit donc être abandonnée. ${ }^{19}$

Les mentalités changent et la mise sur pied d'un programme de prédonation intrahospitalier pour les patients devant subir une chirurgie cardiaque est devenue, en 1994, plus facile. Grâce à la collaboration soutenue et enthousiaste des services transfusionnels de la Société canadienne de la Croix-Rouge et de tout notre personnel hospitalier, un tel programme est en voie d'être instauré à l'Institut de Cardiologie de Montréal. Les difficultés rencontrées ont été rapidement surmontées, sans aucun doute en raison de l'inquiétude omniprésente entourant les risques infectieux des PSH.

En conformité avec les recommandations de la Société canadienne de la Croix-Rouge, les patients dont la sérologie est positive seront exclus de notre programme. ${ }^{4}$ Cette ligne de conduite augmente la sécurité des inventaires et celle du personnel soignant mais, malheureusement, expose ces patients aux risque de pathologies infectieuses autres que celles dont ils sont déjà porteurs. Cette problématique ne peut être dissociée de celle de la non utilisation obligatoire des unités superflues. Si la société dans son ensemble décidait, à l'avenir, que les unités autologues inutilisées pouvaient servir à des fins homologues, le risque qu'un receveur séronégatif soit affecté négativement par la transfusion d'une unité autologue infectée devra être opposé au risque du patient à qui l'on aura refusé la prédonation et qui acquiert une nouvelle pathologie transmise par voie transfusionnelle. ${ }^{20}$ Ces questions devront trouver réponse dans un avenir rapproché.

En conclusion, le don de sang autologue en chirurgie cardiaque est une méthode sécuritaire, efficace et grandement sous-utilisée pour réduire l'exposition aux PSH tout en augmentant la disponibilité des produits sanguins pour ces patients. Quelques interrogations demeurent mais le don autologue, associé aux autres modalités thérapeutiques permettant de diminuer les transfusions après la circulation extracorporelle, devra dorénavant faire par- tie intégrante de la stratégie transfusionnelle de tout centre où se pratique la chirurgie cardiaque.

\section{References}

1 Hardy J-F, Bélisle $S$, Robitaille D. Blood products: when to use them and how to avoid them. Can J Anaesth 1994; 41: R52-61.

2 Goodnough LT, Johnston MFM, Toy PTCY, The Transfusion Medicine Academic Award Group. The variability of transfusion practice in coronary artery bypass surgery. JAMA 1991; 265: 86-90.

3 Forbes JM, Anderson MD, Anderson GF, Bleecker GC, Rossi EC, Moss GS. Blood transfusion costs: a multicenter study. Transfusion 1991; 31: 318-23.

4 Anonymous. Clinical Guide to Transfusion, 3rd Edition. The Canadian Red Cross Society: 1993.

5 Spiess BD. Hemorrhage, coagulation, and transfusion: a risk-benefit analysis. J Cardiothorac Vasc Anesth 1994; 8(Suppl 1): 19-22.

6 Kruskall MS, Glazer EE, Leonard SS, et al. Utilization and effectiveness of a hospital autologous preoperative blood donor program. Transfusion 1986; 26: 335-40.

7 Britton LW, Eastlund DT, Dziuban SW, et al. Predonated autologue blood use in elective cardiac surgery. Ann Thorac Surg 1989; 47: 529-32.

8 Owings DV, Kruskall MS, Thurer RL, Donoval LM. Autologous blood donations prior to elective cardiac surgery. Safety and effect on subsequent blood use. JAMA 1989; 262: 1963-8.

9 Chambers LA, Kruskall MS. Preoperative autologous blood donation. Transfusion Medicine Reviews 1990; 4: 35-46.

10 Spiess BD, Sassetti R, McCarthy RJ, Narbone RF, Tuman $K J$, Ivankovich $A C$. Autologous blood donation: hemodynamics in a high-risk patient population. Transfusion 1992; 32: 17-22.

11 Love TR, Hendren WG, O'Keefe DD, Daggett WM. Transfusion of predonated autologous blood in elective cardiac surgery. Ann Thorac Surg 1987; 43: 508-12.

12 Mann M, Sacks HJ, Goldfinger D. Safety of autologous blood donation prior to elective surgery for a variety of potentially "high-risk" patients. Transfusion 1983; 23: 229-32.

13 Pinkerton $P H$. Autologous blood donation in support of cardiac surgery: a preliminary report on a hospital-based autologous donor program. Can J Anaesth 1994; 41: 1036-40.

14 Hardy J-F, Perrault J, Tremblay N, Robitaille D, Blain $R$, Carrier $M$. The stratification of cardiac surgical procedures according to use of blood products: a retrospective analysis of 1480 cases. Can J Anaesth 1991; 38: 511-7.

15 Axelrod FB, Pepkowitz SH, Goldfinger D. Establishment of a schedule of optimal preoperative collection of autologous blood. Transfusion 1989; 29: 677-80. 
16 Goodnough LT, Rudnick S, Price $T H$, et al. Increased preoperative collection of autologous blood with recombinant human erythropoietin therapy. N Engl J Med 1989; 321: 1163-8.

17 Kyo S, Omoto R, Hirashima K, Eguchi S, Fujita T. Effect of human recombinant erythropoietin on reduction of homologous blood transfusion in open-heart surgery. Circulation 1992; 86(Suppl II): II-413-8.

18 Nonoguchi $H$, Abe K, Komatsu F. Recombinant human erythropoietin for autologous blood donation (Letter). Lancet 1992; 339: 1483.

19 Goodnough LT, Johnston MFM, Ramsey G, et al. Guidelines for transfusion support in patients undergoing coronary artery bypass grafting. Ann Thorac Surg 1990; 50: 675-83.

20 Dzik $W H$, Devarajan $S$. Should autologous blood that tests positive for infectious diseases be used or discarded? A decision analysis approach. Transfusion 1989; 29: 743-45. 